\title{
Long non-coding RNA in pancreatic adenocarcinoma and pancreatic neuroendocrine tumors
}

\author{
Dimitrios Moschovisa, Maria Gazouli ${ }^{b}$, Maria Tzouvalaa, Antonios Vezakisc, George Karamanolis ${ }^{\mathrm{d}}$ \\ Agios Panteleimon General Hospital, Nikea; School of Medicine, National and Kapodistrian University of Athens; \\ Aretaieion University Hospital, National and Kapodistrian University of Athens, Athens, Greece
}

Abstract

\begin{abstract}
Interest in non-coding regions of DNA has been increasing since the mapping of the human genome revealed that human DNA contains far fewer genes encoding proteins than previously expected. However, analysis of the derivatives of DNA transcription (transcriptomics) revealed that the majority of the genetic material is transcribed into non-coding RNA (ncRNA), indicating that these molecules probably provide the functional diversity and complexity of the physiology of the human body that cannot be attributed to the proteins. Of these ncRNA, long ncRNA (lncRNA) have a length greater than 200 nucleotides and share many common components with the coding messenger RNA (mRNA): They are transcribed by RNA polymerase II, comprised of multiple exons and subjected to normal RNA splicing giving RNA products of several kilobases. Scientific data reveal the regulatory role of lncRNA in the control of gene expression during cell development and homeostasis. However, to date, very few lncRNAs have been characterized in depth, and lncRNAs are thought to have a wide range of functions in cellular and developmental processes. These molecules will have the possibility to be used as biomarkers and contribute to the development of targeted therapies. Concerning pancreatic cancer, there are limited data in the literature that correlate the growth of these tumors with deregulation of various $\operatorname{lncRNA}$. We herein review the literature regarding the role of lncRNA as a diagnostic and prognostic biomarker and possible therapeutic target in the neoplasms of the pancreas, particularly pancreatic adenocarcinoma and pancreatic neuroendocrine tumors.
\end{abstract}

Keywords Long non-coding RNA, pancreatic ductal adenocarcinoma, pancreatic neuroendocrine tumors, pancreatic tumors

Ann Gastroenterol 2017; 30 (5): 1-7

\section{Introduction}

According to the World Health Organization classification, the pancreas gives rise to several malignant and benign neoplasms. Ductal adenocarcinoma, which represents the most common

Departments of ${ }^{\mathrm{a}}$ Gastroenterology, Agios Panteleimon General Hospital, Nikea (Dimitrios Moschovis, Maria Tzouvala); ${ }^{\text {Basic Medical }}$ Sciences, Laboratory of Biology, School of Medicine, National and Kapodistrian University of Athens (Maria Gazouli); ${ }^{\text {nd }}$ Department of Surgery, Aretaieion University Hospital, National and Kapodistrian University of Athens (Antonios Vezakis); 'Gastroenterology Unit, $2^{\text {nd }}$ Department of Surgery, Aretaieio University Hospital, National and Kapodistrian University of Athens (George Karamanolis), Greece

\section{Conflict of Interest: None}

Correspondence to: George Karamanolis MD, PhD, Gastroenterology Unit, $2^{\text {nd }}$ Department of Surgery, Aretaieio University Hospital, National and Kapodistrian University of Athens, Athens 11528, Greece, e-mail: georgekaramanolis@yahoo.co.uk

Received 25 March 2017; accepted 5 July 2017; published online 4 August 2017

DOI: https://doi.org/10.20524/aog.2017.0185 type of exocrine carcinoma, accounts for approximately $85 \%$ of pancreatic tumors, while tumors derived from the endocrine pancreas, arising from the cells of the islets, represent $5 \%$ [1].

Pancreatic ductal cell adenocarcinoma (PDAC) is showing an increasing incidence in the developed countries [2-4]. Factors associated with the higher incidence of PDAC are smoking, obesity, diet, diabetes mellitus, chronic pancreatitis, and genetic predisposition in approximately $5-10 \%$ of patients [2,5-8]. Genes found to be involved in the majority of adenocarcinomas are KRAS, CDKN2A, TP53, and SMAD4 [6]. Surgical resection seems to be the method of choice for a possible cure; however, only $10-20 \%$ of patients have potentially resectable pancreatic cancer (PC) at the time of diagnosis $[9,10]$. Following surgery, adjuvant chemotherapy with gemcitabine or 5-fluorouracil is recommended $[10,11]$. FOLFIRINOX and protein-bound paclitaxel (nab-paclitaxel) chemotherapy was found to be more effective than gemcitabine, but with notable side effects [12]. Several ongoing late-phase trials are currently evaluating a number of therapeutic targets (inhibition of growth factor receptors: Epidermal growth factor receptor, platelet-derived growth factor receptors, vascular endothelial growth factor receptor, insulin-like growth factor receptor-1, tyrosine kinase inhibitors, MEK1/2, mTOR blockade and PI3K and HER2-neu 
pathway inhibitors); their results will become available over the next 1-2 years [13].

Pancreatic neuroendocrine tumors (PNET) occur in two distinct epidemiologic groups: Sporadic tumors and the tumors that affect kindreds with the multiple endocrine neoplasia type 1 syndrome (MEN1) in a pattern of autosomal dominant inheritance [14]. Gastrinoma and insulinoma are the most frequent lesions, with equal incidences [15], while nonfunctional tumors account for $14-48 \%$ of neoplasms of the endocrine pancreas $[16,17]$. No well-established environmental factors are associated with the development of neoplasms of the endocrine pancreas [18]. Genes frequently mutated in these patients include $D A X X, m T O R$ and ATRX [19]. Other than surgery for functioning tumors [20,21], the somatostatin analogs, such as octreotide, can reduce the excessive production of these hormones [22]. Targeted therapy with everolimus or sunitinib can control symptoms and decelerate the progression of the disease $[19,23]$.

\section{Materials and methods}

An extensive English-language literature search was conducted using PubMed to identify original studies and review articles, using as keywords "pancreatic tumors", "prognostic factors", "biomarkers", "pancreatic adenocarcinoma", "pancreatic neuroendocrine tumors", and "long non-coding RNA".

\section{Discussion}

\section{Non-coding RNAs (ncRNAs)}

NcRNA can be classified into two groups according on their size: The group of short ncRNA, which are less than 200 nucleotides (nt) in length and include micro RNA (miRNA), piwi-interacting RNA (piRNA), ribosomal RNA (rRNA), transfer RNA (tRNA), small nuclear RNA (snRNA), small nucleolar RNA (snoRNA), and telomere-associated RN [24]; and the other group of lncRNA, which contain $200 \mathrm{nt}$ or more [25]. Based on their proximity to protein-coding genes, lncRNA are classified into five types: Sense, antisense, bidirectional, intronic and intergenic [26,27].

\section{Long ncRNA (IncRNA)}

Initially, lncRNA was considered to be "transcriptional noise" without any biological function. However, nowadays it has been demonstrated that IncRNA, interacting with DNA, RNA and transcription factors, participates in a series of biological processes, such DNA methylation, histone modification, and chromatin remodeling. In this way, it manages the expression status of target genes controlling the cell cycle [28-30], the induction of angiogenesis, the promotion of metastasis, and the evasion of tumor suppressors $[31,32,49,50]$, making them important "players" in the diagnosis and treatment of malignant tumors $[33,34]$.
Different studies have reported that the primary sequences of lncRNA show very little conservation, in contrast with their secondary and tertiary structures, which are highly conserved and might be potentially related to their biological functions [35-37]. Recent studies have shown that a variety of lncRNA can be used as tumor markers [38-40].

All lncRNA execute their functions through five main means: As signals, decoys, guides, enhancers, or scaffolds [41]. In these ways lncRNA regulates the progress of various biologic processes and the expression of various genes controlling the homeostasis of the cell cycle.

\section{LncRNAs and PDAC}

In recent years, the regulatory roles of lncRNA in the etiology of several diseases have attracted increasing interest. The strongest relation at present is with cancer $[42,43]$. Altered expression of lncRNA has been documented in different human cancers, increasing the interest in their role as biomarkers for diagnosis and prognosis, as well as potential therapeutic targets for these types of diseases $[44,45]$. Abnormal lncRNA expression in many cancers might be a major cause of oncogenesis. These cancers can be distinguished according to their altered lncRNA expression $[25,46]$.

PDAC is known for its aggressiveness and lack of effective therapeutic options. Thus, there is an urgent need to explore novel approaches to the diagnosis and treatment of this dismal disease.

Genetic alterations associated with PC progression include eventssuchasmutationsinK-RAS(approx.90\%)andoverexpression of HER-2/neu. Inactivation of the p16/CDKN2A (approx. 75\%) tumor suppressor gene often occurs at later stages, followed by loss of p53 (approx. 65\%), SMAD4 deregulation (approx. 50\%), and BRCA2 signaling pathways that facilitate deregulation of cellcycle control, invasion and metastasis. Despite these findings, the alterations of oncogenes and tumor suppressor genes have not yet led to solutions for therapeutic interventions [47].

In recent years, it has been proposed that epigenetic events may also play an important role in the development and maintenance of PC. LncRNAs are already known as important epigenetic regulators that have a role in diverse cellular processes including cell proliferation, development, differentiation, apoptosis and therefore oncogenesis [48].

Of the lncRNAs normally expressed on pancreatic tissue, some were significantly upregulated and others significantly downregulated in PDAC tissue samples.

\section{H19}

H19 is a maternally expressed and paternally imprinted $2.3 \mathrm{~kb}$ gene; it resides close to the telomeric region of chromosome $11 \mathrm{p} 15.5$ and is mainly localized in cytoplasm [49]. H19 is known to respond to various stress conditions, such as reduced p53 and hypoxia-inducing epithelial-to-mesenchymal transition (EMT), cell invasion and extravasation/migration, by activation mechanisms of cell survival [50]. 
According to a model suggested by Zipori et al [51,52], p53, a well-known genome keeper, interplays with H19 in cellular homeostasis. p53 is an H19 suppressor. During stress conditions prorogated by downregulation of p53, cells can respond to this by upregulation of $H 19$.

Another common stress condition in tumors is hypoxia. In addition to the abnormal vascularization commonly affecting their essential nutrient and gas exchange [53], the high proliferation rate of cancer cells impedes their access to normal blood supply, prorogating hypoxia [54] that induces $H 19$ transcription [55] when p53 is null or impaired [56]. Hypoxia-inducible factor1a (HIF-1a) mediates H19 induction during hypoxia when p53 is impaired, possibly because of the loss of p53's inhibitory effect on HIF-1a. Nuclear localization of p53 is essential for H19 inhibition [56]. H19 promotes direct tumor metastasis by upregulation of EMT, favoring the dedifferentiation of epithelial cells that become able to disseminate and migrate through the extracellular matrix (ECM), ensuring the intravasation into blood vessels that will carry them to a distal site. Once extravased to the secondary site, the tumor cell re-differentiates, through the reverse mechanism of mesenchymal-to-epithelial transition, and then proliferates and colonizes, becoming a secondary tumor [57]. In one study, it was found that $\mathrm{H} 19$ was overexpressed in PDAC compared with adjacent normal tissues. Upregulation of H19 expression was also notable in primary tumors that subsequently metastasized, compared to those that did not [58]. In the same study, downregulation of H19 impaired PDAC progression. H19 has been demonstrated to promote PDAC cell invasion and migration, mainly by increasing HMGA2-mediated EMT through antagonizing let-7 [58]. The role of H19 in the oncogenesis and metastasis of PDAC is schematically described in Fig. 1.

A phase I/IIa study in patients with unresectable PC investigated the efficacy of intratumoral administration of the plasmid diphtheria toxin A (DTA)-H19 composed of the H19 gene regulatory sequences. DTA-H19 is a double-stranded DNA plasmid that carries the gene for the DTA chain under the regulation of the $H 19$ promoter sequence. This plasmid embodies a targeted therapy approach, in that the plasmid enters all dividing cells, but the DTA expression is triggered by the presence of $\mathrm{H} 19$ transcription factors found only in tumor cells, thus destroying the tumor without affecting normal cells [59].

Additionally, studies have demonstrated that the efficacy of PC treatment should be improved by DNA-based therapy controlled by $\mathrm{H} 19$ gene sequences, either alone or in combination with gemcitabine [60].

\section{LncRNA HOXA transcript at the distal tip (HOTTIP)}

The HOTTIP lncRNA, located at the 5 ' end of the HOXA cluster, was recently functionally characterized [61]. The activity of HOTTIP in the regulation of proliferation, invasion, and chemoresistance of $\mathrm{PC}$ is a result of its interaction with the WDR5/MLL complex, which enhances histone H3 lysine 4 trimethylation to activate the expression of multiple 5' HOXA genes [61].

It has been demonstrated that targeted silencing of HOTTIP impairs the mechanisms of proliferation, invasion, and EMT capability. In the same study, HOTTIP was found to be upregulated in most PDAC tissues compared with adjacent non-tumor tissues [62]. Moreover, HOTTIP seems to have a prognostic value for gemcitabine chemoresistance in PC cells. Furthermore, HOXA13, a gene also involved in the progression of PDAC, is located in contiguity with HOTTIP and is one of its significant targets. In a study that investigated chronic pancreatitis tissues, 27 lncRNA, including HOTTIP, were upregulated more than 10-fold in PDAC compared with these chronic pancreatitis tissues [62].

From the five splice variants of HOTTIP, HOTTIP-005 expression in a normal cell line and six PC cell lines was examined [63]. All PC cell lines had higher HOTTIP-005 expression compared with the normal cells. Univariate analysis of overall survival revealed that TNM stage, early recurrence and HOTTIP-005 level expression were independent prognostic indicators for the overall survival of patients with PC; the median time of survival in the high- and low-level HOTTIP-005 expression subgroups was 14 and 45 months, respectively. These results indicate that increased HOTTIP-005 expression is a poor prognostic factor for patients with PC [63].

\section{Metastasis associated lung adenocarcinoma transcript 1 (MALAT1)}

MALAT1 is more than $8000 \mathrm{nt}$ in length and localized on chromosome 11q13 [64].

It is significantly over expressed in many cancer types [65-67] and has been shown to promote tumor cell proliferation, invasion and metastasis through polycomb repressive complex 2 (PRC2) [68,85], the Wnt pathway [69], the ERK/MAPK pathway [70] and the SFPQ/PTBP2 complex [71], which is involved in tumor growth. MALAT1 can also facilitate EMT by downregulating the expression of E-cadherin and upregulating the expression of $\mathrm{N}$-cadherin and fibronectin to enhance transforming growth factor-s $\beta$-induced EMT [72]. Thus, high

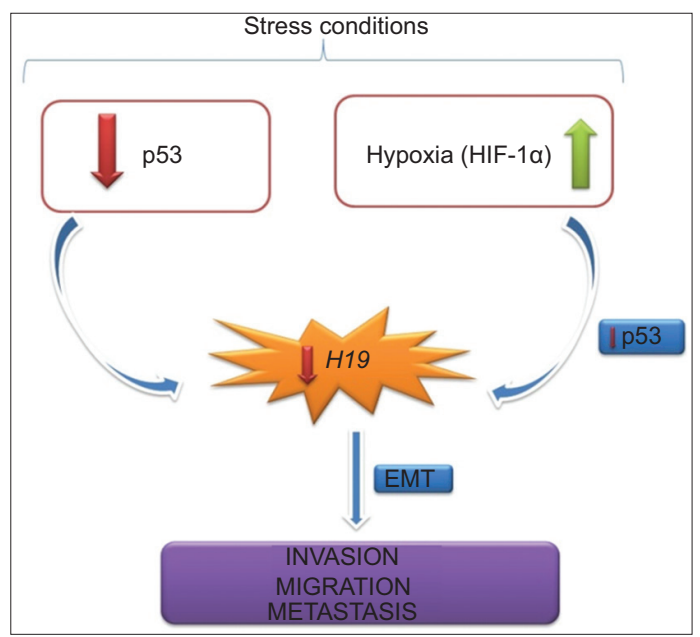

Figure 1 Role of $H 19$ in pancreatic adenocarcinoma HIF-1 $\alpha$, hypoxia-inducible factor-1 $\alpha$; EMT, epithelial-to-mesenchymal transition 
levels of MALAT1 expression may be related to tumor prognosis, indicating its potential use as a biomarker of cancer.

Another analysis indicated that overexpression of MALAT1, additionally to the tumor location and nerve invasion, was an independent predictive factor for overall survival in patients with PDAC. The level of MALAT1 was significantly higher in PDAC compared to the adjacent normal pancreatic tissues. Furthermore, MALAT1 expression level showed significant correlation with TNM stage. Higher MALAT1 expression was associated with poorer disease-free survival in patients with PC [73]. In contrast to the previous study by Liu et al [73], another study did not confirm the MALAT1 upregulation in PDAC [74]. A meta-analysis carried out in 2016 found that in PC there is a significant association between MALAT1 overexpression and TNM stage, as well as with distant metastasis and cancer prognosis but not with lymph node metastasis [75].

\section{HOX antisense intergenic RNA (HOTAIR)}

HOTAIR is a lncRNA localized on chromosome 12q12.13 at a boundary in the HOXC gene cluster. The activity of HOTAIR is due to its interaction with PRC2 (EZH2, SUZ12 and EED), which enhances H3K27 trimethylation to decrease the expression of multiple genes involved in cell proliferation and metastasis [76]. Studies showed that HOTAIR is more highly expressed in advanced tumors compared to early-stage tumors and is overexpressed in pancreatic tumors compared to the normal pancreas [77-79]. Targeted silencing of HOTAIR in PC cells decreased cell proliferation, inhibited cell-cycle progression, and induced apoptosis [76].

HOTAIR is also highly expressed in the metastatic sites of cancer that have poor outcomes in patients undergoing chemotherapy or radiotherapy [80]. In another study, HOTAIR was more highly expressed in locally advanced tumors $\left(\mathrm{T}_{3}\right)$ compared with tumors only detected in the pancreas $\left(\mathrm{T}_{2}\right)$, and more highly expressed in tumors involving regional lymph nodes $\left(\mathrm{N}_{1}\right)$ compared with tumors localized only in the pancreas $\left(\mathrm{N}_{0}\right)$ [78]. In this same study, a survival analysis showed that low HOTAIR expression $(<85 \%)$ was associated with significantly increased overall survival compared to high HOTAIR expression in patients with PC. HOTAIR levels and N stage are strongly correlated with overall patient survival [78].

A study by Chang's group uncovered a new mechanism whereby HOTAIR leads to transcriptional silencing of a distant chromosomal region through epigenetic regulation [81]. Enforced HOTAIR expression in epithelial cancer cells was found to increase invasive and metastatic abilities and reprogram the PRC2 occupancy pattern to resemble embryonic fibroblasts [45].

\section{AF339813}

AF339813 is regulated positively by NUF2. A study that investigated NUF2 expression in cancer tissues from patients with PC found it to be significantly higher than that in adjacent normal tissues. It was also demonstrated that knockdown of AF339813 in PC cells significantly reduced cell proliferation and promoted apoptosis [82].
Growth arrest-specific 5 (Gas5)

The expression level of gas 5 was found to be significantly decreased in PC compared with normal tissues. Overexpression of gas 5 in these cells inhibits cell proliferation, whereas gas5 inhibition induces a significant decrease in the $G_{0} / G_{1}$ phase and an increase in the $S$ phase. It was also demonstrated that gas5 negatively regulates CDK6 (cyclin-dependent kinase 6) expression in vitro and in vivo. Knockdown of CDK6 partially reduce gas5-induced cell proliferation [83].

\section{ENST00000480739}

A study of 35 PDAC noted that lncRNA ENST00000480739 expression was remarkably lower in PDAC tissues than in adjacent normal tissues. Results revealed that ENST00000480739 expression was negatively correlated with tumor size and with lymph node involvement by modulating HIF-1a. No significant correlation between ENST00000480739 expression and age, sex, diameter or degree of differentiation was proved [84].

\section{Highly upregulated in liver cancer (HULC)}

HULC is a cancer-related lncRNA, residing on chromosome 6 p24.3 and located in the cytoplasm. Overexpression of HULC is observed in a group of advanced staged and metastatic pancreatic tumors. This expression profile is related to the promotion of cell proliferation in vitro [85].

\section{LncRNAs and PNET}

The most common location of NETs is in the intestines, pancreas, and lung; they can be classified as functional (hormone secreting) or nonfunctional (non-hormone secreting) NETs [86]. There are few studies in the literature relating to ncRNA and neuroendocrine malignancies. Data from a study by Roldo et al showed that a common pattern of miRNA expression distinguishes any tumor type from normal pancreas, suggesting that this set of miRNA might be involved in pancreatic tumor genesis. Specifically, the expression of miR103 and miR-107, together with a lack of expression of miR-155, characterize neoplasmatic but not normal pancreatic tissue. miR-204 is primarily expressed in insulinomas and correlates with the immunohistochemical expression of insulin, while overexpression of miR-21 is strongly associated with both a high Ki67 proliferation index and the presence of liver metastasis [87].

There are only scanty data in the literature concerning the role of IncRNA in PNET. The most studied correlation concerns the MEN1 gene-encoding "menin" protein in PNET, but how this initiates tumorigenesis is not yet well understood. In a study by Modali et al [88], it was found that lncRNA Meg3 (maternally expressed gene) has tumor-suppressor activity in PNET cells. Menin produced in PNET activates Meg3. Meg3 overexpression in insulinoma cells delays cell proliferation by downregulation of the expression of the proto-oncogene c-Met and these cells show significantly reduced cell migration and 
invasion. It was also found that Meg3 and c-MET levels are reciprocally correlated, not only with MEN1-associated PNET, but also with human sporadic insulinomas.

These observations provide a strong basis for the investigation of Meg3 activation and c-MET suppression as novel therapeutic approaches for the treatment of pancreatic neuroendocrine tumors and possibly for the prevention of metastasis.

\section{LncRNA as biomarkers and therapeutic target in PC}

An ideal biomarker is defined as the marker that offers the advantages of early detection, high specificity, sufficient sensitivity, robustness and minimal invasion. Many lncRNA are emerging as new candidate biomarkers of cancer, having regulatory functions in both oncogenic and tumor-suppressive pathways, such as the $\mathrm{p} 53, \mathrm{MYC}$, and NF- $\mathrm{B}$ pathways $[89,90]$. The concept of lncRNA as disease markers is strongly related to the discovery that lncRNA demonstrate extreme molecular stability. Additionally, as opposed to mRNA, the lncRNA are functional molecules and their expression level may be an improved marker of the disease. Furthermore, the highly specific expression patterns of lncRNA can be used for accurate disease diagnostics and classification [91]. To date, there is no specific biomarker for the early diagnosis of PC. CA 19-9 is used as biomarker in general clinical practice, although its level has no utility as a screening tool in asymptomatic patients. Even in symptomatic patients where there is suspicion of PC, elevated CA $19-9$ is a poor predictor, with a predictive value of $0.5-0.9 \%$ [92].

The importance of lncRNA as a regulatory molecule of the cell cycle makes it an attractive therapeutic target; thus, there is a need to find methods for modulating lncRNA. For this reason, short DNA sequences complementary to the RNA of interest, called allele-specific oligonucleotides (ASO), have been recruited. These oligonucleotides work by hybridizing to the RNA, which then blocks the action of the RNA. So far, two ASO have been approved as drugs by the Food and Drug Administration (FDA) of the United States: Fomivirsen, which is used to treat cytomegalovirus retinitis [93], and mipomersen, which is used to treat homozygous familial hypercholesterolemia [94]. ASO act by entering the nucleus and knocking down nuclear lncRNA [95].

A major challenge to working on lncRNA is that the molecular mechanisms underlying their functions are not yet fully understood. Further insight into the biological significance and functioning of IncRNA will require additional studies to be conducted, which may lead to the discovery of yet more mechanisms of action. A further confounding factor in our understanding of lncRNA is that lncRNA may have more than one mechanism of action to confer transcriptional activation or repression of their target genes. The characteristics of deregulated lncRNA described in PDAC and PNET are summarized in Table 1.

\section{Concluding remarks}

Expansion of our knowledge of the role of lncRNA in oncogenesis will help improve the diagnosis and develop
Table 1 Deregulated IncRNA in PDAC and PNET

\begin{tabular}{llll}
\hline LncRNA & Deregulation & Neoplasm & Sample origin \\
\hline H19 & up & PDAC & Pancreatic tissue \\
HOTTIP & up & PDAC & Pancreatic tissue \\
MALAT1 & up & PDAC & Pancreatic tissue \\
HOTAIR & up & PDAC & Pancreatic tissue \\
AF339813 & up & PDAC & Pancreatic tissue \\
Gas5 & down & PDAC & Pancreatic tissue \\
ENST00000480739 & down & PDAC & Pancreatic tissue \\
HULC & up & PDAC & Pancreatic tissue \\
Meg3 & up & PNET & Pancreatic tissue \\
\hline
\end{tabular}

LncRNA, long non-coding RNA; PDAC, pancreatic ductal cell

adenocarcinoma; PNET, pancreatic neuroendocrine tumors

therapeutic options for PDAC and PNET. Taking into account the limited number of studies regarding the role of lncRNA in these neoplasms, more studies will be needed in the future to demonstrate the importance of lncRNA in tumor initiation, invasion and metastasis, as well as their utility as therapeutic targets. Moreover, considering that until now lncRNA have been detected in pathological pancreatic tissues, their investigation in "easy access" materials like blood in the future will make them even more attractive and less invasive biomarkers. The potential therapeutic options for modulating lncRNA include the form of ASO, as well as other technologies that may arise. LncRNA-based therapies could become an important strategy deserving consideration.

\section{References}

1. Klimstra DS. Nonductal neoplasms of the pancreas. Mod Pathol 2007;20 Suppl 1:S94-S112.

2. Ryan DP, Hong TS, Bardeesy N. Pancreatic adenocarcinoma. N Engl J Med 2014;371:1039-1049.

3. He Y, Zheng R, Li D, Zeng H, Zhang S, Chen W. Pancreatic cancer incidence and mortality patterns in China, 2011. Chin J Cancer Res 2015;27:29-37.

4. Siegel R, Naishadham D, Jemal A. Cancer statistics, 2013. CA Cancer J Clin 2013;63:11-30.

5. Bond-Smith G, Banga N, Hammond TM, Imber CJ. Pancreatic adenocarcinoma. BMJ 2012;344:e2476.

6. Wolfgang CL, Herman JM, Laheru DA, et al. Recent progress in pancreatic cancer. CA Cancer J Clin 2013;63:318-348.

7. Bosetti C, Lucenteforte E, Silverman DT, et al. Cigarette smoking and pancreatic cancer: An analysis from the International Pancreatic Cancer Case-Control Consortium (Panc4). Ann Oncol 2012;23:1880-1888.

8. Peters ML, Tseng JF, Miksad RA. Genetic testing in pancreatic ductal adenocarcinoma: Implications for prevention and treatment. Clin Ther 2016;38:1622-1635.

9. Stathis A, Moore MJ. Advanced pancreatic carcinoma: Current treatment and future challenges. Nat Rev Clin Oncol 2010;7:163-172.

10. Seufferlein T, Bachet JB, Van Cutsem E, Rougier P; ESMO Guidelines Working Group. Pancreatic adenocarcinoma: ESMOESDO Clinical Practice Guidelines for diagnosis, treatment and 
follow-up. Ann Oncol 2012;23 Suppl 7:vii33-vii40.

11. Vincent A, Herman J, Schulick R, Hruban RH, Goggins M. Pancreatic cancer. Lancet 2011;378:607-620.

12. Borazanci E, Von Hoff DD. Nab-paclitaxel and gemcitabine for the treatment of patients with metastatic pancreatic cancer. Expert Rev Gastroenterol Hepatol 2014;8:739-747.

13. Mosquera C, Maglic D, Zervos EE. Molecular targeted therapy for pancreatic adenocarcinoma: A review of completed and ongoing late phase clinical trials. Cancer Genet 2016;209:567-581.

14. Norton JA, Levin B, Jensen RT. Cancer of the endocrine system. In: DeVita VT, Hellman S, Rosenberg SA (editors). Cancer: Principles and Practice of Oncology, $4^{\text {th }}$ ed. Lippincott-Raven: Philadelphia, Pa; 1993, pp. 1333-1435.

15. Jansen RT, Norton JA. Endocrine tumors of pancreas. In: Feldman $M$, Scharschmidt BF, Sleisenger $M$ (editors). Sleisenger and Fordtran's Gastrointestinal and Liver Disease, $6^{\text {th }}$ ed. WB Saunders: Philadelphia, Pa; 1998, pp. 871-894.

16. Ericcson B, Oberg k. PPomas and son functioning endoscrine pancreatic tumors: clinical presentation, diagnosis and advances in management. In: Mignon M, Jensen RT (editors). Endocrine Tumors of the Pancreas. Frontiers of Gastrointestinal Research, Vol 23. Karger: Basel, Switzerland; 1995, p. 208.

17. Phan GQ, Yeo CJ, Hruban RH, Lillemoe KD, Pitt HA, Cameron JL. Surgical experience with pancreatic and peripancreatic neuroendocrine tumors: Review of 125 patients. J Gastrointest Surg 1998;2:472-482.

18. Nakeeb A, Lillemoe KD, Yeo CJ. Neoplasms of the exocrine pancreas. In: Greenfield LJ, Mullholand MW, Oldham KT (editors). Surgery: Scientific Principles and Practice, $3^{\text {rd }}$ ed. LippincottRaven: Philadelphia Pa; 2001, pp. 558-599.

19. Burns WR, Edil BH. Neuroendocrine pancreatic tumors: Guidelines for management and update. Curr Treat Options Oncol 2012;13:24-34.

20. Falconi M, Bartsch DK, Eriksson B, et al; Barcelona Consensus Conference participants. ENETS Consensus Guidelines for the management of patients with digestive neuroendocrine neoplasms of the digestive system: Well-differentiated pancreatic nonfunctioning tumors. Neuroendocrinology 2012;95:120-134.

21. Jensen RT, Cadiot G, Brandi ML, et al; Barcelona Consensus Conference participants. ENETS Consensus Guidelines for the management of patients with digestive neuroendocrine neoplasms: Functional pancreatic endocrine tumor syndromes. Neuroendocrinology 2012;95:98-119.

22. Öberg K, Knigge U, Kwekkeboom D, Perren A; ESMO Guidelines Working Group. Neuroendocrine gastro-entero-pancreatic tumors: ESMO Clinical Practice Guidelines for diagnosis, treatment and follow-up. Ann Oncol 2012;23 Suppl 7:vii124-vii130.

23. Yao JC, Shah MH, Ito T, et al; RAD001 in Advanced Neuroendocrine Tumors, Third Trial (RADIANT-3) Study Group. Everolimus for advanced pancreatic neuroendocrine tumors. $N$ Engl J Med 2011;364:514-523.

24. Bushati N, Cohen SM. microRNA functions. Annu Rev Cell Dev Biol 2007;23:175-205.

25. Esteller M. Non-coding RNAs in human disease. Nat Rev Genet 2011;12:861-874.

26. Mercer TR, Dinger ME, Mattick JS. Long non-coding RNAs: Insights into functions. Nat Rev Genet 2009;10:155-159.

27. Mattick JS. The genetic signatures of noncoding RNAs. PLoS Genet 2009;5:e1000459.

28. Chen LL, Carmichael GG. Decoding the function of nuclear long non-coding RNAs. Curr Opin Cell Biol 2010;22:357-364.

29. Kino T, Hurt DE, Ichijo T, Nader N, Chrousos GP. Noncoding RNA gas5 is a growth arrest- and starvation-associated repressor of the glucocorticoid receptor. Sci Signal 2010;3:ra8.

30. Mourtada-Maarabouni M, Pickard MR, Hedge VL, Farzaneh F, Williams GT. GAS5, a non-protein-coding RNA, controls apoptosis and is downregulated in breast cancer. Oncogene 2009;28:195-208.

31. Gutschner T, Diederichs S. The hallmarks of cancer: A long noncoding RNA point of view. RNA Biol 2012;9:703-719.

32. Brunner AL, Beck AH, Edris B, et al. Transcriptional profiling of long non-coding RNAs and novel transcribed regions across a diverse panel of archived human cancers. Genome Biol 2012;13:R75.

33. Gong Z, Zhang S, Zeng Z, et al. LOC401317, a p53-regulated long non-coding RNA, inhibits cell proliferation and induces apoptosis in the nasopharyngeal carcinoma cell line HNE2. PLoS One 2014;9:e110674.

34. Gong Z, Zhang S, Zhang W, et al. Long non-coding RNAs in cancer. Sci China Life Sci 2012;55:1120-1124.

35. Mercer TR, Mattick JS. Structure and function of long noncoding RNAs in epigenetic regulation. Nat Struct Mol Biol 2013;20:300-307.

36. Johnsson P, Lipovich L, Grandér D, Morris KV. Evolutionary conservation of long non-coding RNAs; sequence, structure, function. Biochim Biophys Acta 2014;1840:1063-1071.

37. Novikova IV, Hennelly SP, Sanbonmatsu KY. Structural architecture of the human long non-coding RNA, steroid receptor RNA activator. Nucleic Acids Res 2012;40:5034-5051.

38. Bo H, Gong Z, Zhang W, et al. Upregulated long non-coding RNA AFAP1-AS1 expression is associated with progression and poor prognosis of nasopharyngeal carcinoma. Oncotarget 2015;6:20404-20418.

39. Zeng Z, Bo H, Gong Z, et al. AFAP1-AS1, a long noncoding RNA upregulated in lung cancer and promotes invasion and metastasis. Tumour Biol 2016;37:729-737.

40. Zhang W, Huang C, Gong Z, et al. Expression of LINC00312, a long intergenic non-coding RNA, is negatively correlated with tumor size but positively correlated with lymph node metastasis in nasopharyngeal carcinoma. J Mol Histol 2013;44:545-554.

41. Wang KC, Chang HY. Molecular mechanisms of long noncoding RNAs. Mol Cell 2011;43:904-914.

42. Gibb EA, Brown CJ, Lam WL. The functional role of long noncoding RNA in human carcinomas. Mol Cancer 2011;10:38.

43. Prensner JR, Chinnaiyan AM. The emergence of lncRNAs in cancer biology. Cancer Discov 2011;1:391-407.

44. Reis EM, Nakaya HI, Louro R, et al. Antisense intronic non-coding RNA levels correlate to the degree of tumor differentiation in prostate cancer. Oncogene 2004;23:6684-6692.

45. Gupta RA, Shah N, Wang KC, et al. Long non-coding RNA HOTAIR reprograms chromatin state to promote cancer metastasis. Nature 2010;464:1071-1076.

46. Perez DS, Hoage TR, Pritchett JR, et al. Long, abundantly expressed non-coding transcripts are altered in cancer. Hum Mol Genet 2008; 17:642-655.

47. Kanda M, Matthaei $\mathrm{H}, \mathrm{Wu}$ J, et al. Presence of somatic mutations in most early-stage pancreatic intraepithelial neoplasia. Gastroenterology 2012;142:730-733.

48. McCleary-Wheeler AL, Lomberk GA, Weiss FU, et al. Insights into the epigenetic mechanisms controlling pancreatic carcinogenesis. Cancer Lett 2013;328:212-221.

49. Matouk I, Raveh E, Ohana P, et al. The increasing complexity of the oncofetal h19 gene locus: Functional dissection and therapeutic intervention. Int J Mol Sci 2013;14:4298-4316.

50. Raveh E, Matouk IJ, Gilon M, Hochberg A. The H19 Long noncoding RNA in cancer initiation, progression and metastasis - a proposed unifying theory. Mol Cancer 2015;14:184.

51. Shoshani O, Massalha H, Shani N, et al. Polyploidization of murine mesenchymal cells is associated with suppression of the long noncoding RNA H19 and reduced tumorigenicity. Cancer Res 2012;72:6403-6413.

52. Ravid O, Shoshani O, Sela M, et al. Relative genomic stability of adipose tissue derived mesenchymal stem cells: Analysis of ploidy, H19 long non-coding RNA and p53 activity. Stem Cell Res Ther 2014;5:139. 
53. Vaupel P, Harrison L. Tumor hypoxia: Causative factors, compensatory mechanisms, and cellular response. Oncologist 2004;9 Suppl 5:4-9.

54. Vaupel P, Mayer A. Hypoxia in cancer: Significance and impact on clinical outcome. Cancer Metastasis Rev 2007;26:225-239.

55. Matouk IJ, DeGroot N, Mezan S, et al. The H19 non-coding RNA is essential for human tumor growth. PLoS One 2007;2:e845.

56. Matouk IJ, Mezan S, Mizrahi A, et al. The oncofetal H19 RNA connection: Hypoxia, p53 and cancer. Biochim Biophys Acta 2010; 1803:443-451.

57. Vanharanta S, Massagué J. Origins of metastatic traits. Cancer Cell 2013;24:410-421.

58. Ma C, Nong K, Zhu H, et al. H19 promotes pancreatic cancer metastasis by derepressing let-7's suppression on its target HMGA2-mediated EMT. Tumour Biol 2014;35:9163-9169.

59. P19 Phase 12a DTA-H19 in patients with unresectable pancreatic cancer. ClinicalTrials.gov U.S. National Institutes of Health. 2009-11-09.

60. Sorin V, Ohana P, Gallula J, et al. H19-promoter-targeted therapy combined with gemcitabine in the treatment of pancreatic cancer. ISRN Oncol 2012;2012:351750.

61. Wang KC, Yang YW, Liu B, et al. A long noncoding RNA maintains active chromatin to coordinate homeotic gene expression. Nature 2011;472:120-124.

62. Li Z, Zhao X, Zhou Y, et al. The long non-coding RNA HOTTIP promotes progression and gemcitabine resistance by regulating HOXA13 in pancreatic cancer. J Transl Med 2015;13:84.

63. Wang Y, Li Z, Zheng S, et al. Expression profile of long noncoding RNAs in pancreatic cancer and their clinical significance as biomarkers. Oncotarget 2015;6:35684-35698.

64. Ji P, Diederichs S, Wang W, et al. MALAT-1, a novel noncoding RNA, and thymosin beta 4 predict metastasis and survival in earlystage non-small cell lung cancer. Oncogene 2003;22:8031-8041.

65. Zhang HM, Yang FQ, Chen SJ, Che J, Zheng JH. Upregulation of long non-coding RNA MALAT1 correlates with tumor progression and poor prognosis in clear cell renal cell carcinoma. Tumour Biol 2015;36:2947-2955.

66. Cho SF, Chang YC, Chang CS, et al. MALAT1 long non-coding RNA is overexpressed in multiple myeloma and may serve as a marker to predict disease progression. BMC Cancer 2014;14:809.

67. Zheng HT, Shi DB, Wang YW, et al. High expression of lncRNA MALAT1 suggests a biomarker of poor prognosis in colorectal cancer. Int J Clin Exp Pathol 2014;7:3174-3181.

68. Yang L, Lin C, Liu W, et al. ncRNA- and Pc2 methylationdependent gene relocation between nuclear structures mediates gene activation programs. Cell 2011;147:773-788.

69. Hirata H, Hinoda Y, Shahryari V, et al. Long Noncoding RNA MALAT1 Promotes Aggressive Renal Cell Carcinoma through Ezh2 and Interacts with miR-205. Cancer Res 2015;75:1322-1331.

70. Wu XS, Wang XA, Wu WG, et al. MALAT1 promotes the proliferation and metastasis of gallbladder cancer cells by activating the ERK/MAPK pathway. Cancer Biol Ther 2014;15:806-814.

71. Ji Q, Zhang L, Liu X, et al. Long non-coding RNA MALAT1 promotes tumour growth and metastasis in colorectal cancer through binding to SFPQ and releasing oncogene PTBP2 from SFPQ/PTBP2 complex. Br J Cancer 2014;111:736-748.

72. Fan Y, Shen B, Tan M, et al. TGF- $\beta$-induced upregulation of malat1 promotes bladder cancer metastasis by associating with suz12. Clin Cancer Res 2014;20:1531-1541.

73. Liu JH, Chen G, Dang YW, Li CJ, Luo DZ. Expression and prognostic significance of lncRNA MALAT1 in pancreatic cancer tissues. Asian Pac J Cancer Prev 2014;15:2971-2977.

74. Müller S, Raulefs S, Bruns P, et al. Next-generation sequencing reveals novel differentially regulated mRNAs, IncRNAs, miRNAs, sdRNAs and a piRNA in pancreatic cancer. Mol Cancer 2015;14:94.

75. Wang Y, Xue D, Li Y, et al. The long noncoding RNA MALAT-1 is a novel biomarker in various cancers: A meta-analysis based on the GEO database and literature. J Cancer 2016;7:991-1001.

76. Kim K, Jutooru I, Chadalapaka G, et al. HOTAIR is a negative prognostic factor and exhibits pro-oncogenic activity in pancreatic cancer. Oncogene 2013;32:1616-1625.

77. Stratford JK, Bentrem DJ, Anderson JM, et al. A six-gene signature predicts survival of patients with localized pancreatic ductal adenocarcinoma. PLoS Med 2010;7:e1000307.

78. Badea L, Herlea V, Dima SO, Dumitrascu T, Popescu I. Combined gene expression analysis of whole-tissue and microdissected pancreatic ductal adenocarcinoma identifies genes specifically overexpressed in tumor epithelia. Hepatogastroenterology 2008;55:2016-2027.

79. Collisson EA, Sadanandam A, Olson P, et al. Subtypes of pancreatic ductal adenocarcinoma and their differing responses to therapy. Nat Med 2011;17:500-503.

80. Khalil AM, Guttman M, Huarte M, et al. Many human large intergenic noncoding RNAs associate with chromatin-modifying complexes and affect gene expression. Proc Natl Acad Sci U S A 2009;106:11667-11672.

81. Rinn JL, Kertesz M, Wang JK, et al. Functional demarcation of active and silent chromatin domains in human HOX loci by noncoding RNAs. Cell 2007;129:1311-1323.

82. Hu P, Shangguan J, Zhang L. Downregulation of NUF2 inhibits tumor growth and induces apoptosis by regulating lncRNA AF339813. Int J Clin Exp Pathol 2015;8:2638-2648.

83. Lu X, Fang Y, Wang Z, et al. Downregulation of gas5 increases pancreatic cancer cell proliferation by regulating CDK6. Cell Tissue Res 2013;354:891-896.

84. Sun YW, Chen YF, Li J, et al. A novel long non-coding RNA ENST00000480739 suppresses tumour cell invasion by regulating OS-9 and HIF-1 $\alpha$ in pancreatic ductal adenocarcinoma. $\mathrm{Br} \mathrm{J}$ Cancer 2014;111:2131-2141.

85. Huang C, Yu W, Wang Q, et al. Increased expression of the lncRNA PVT1 is associated with poor prognosis in pancreatic cancer patients. Minerva Med 2015;106:143-149.

86. Chen X, Liang H, Zhang J, Zen K, Zhang CY. Horizontal transfer of microRNAs: Molecular mechanisms and clinical applications. Protein Cell 2012;3:28-37.

87. Kentwell J, Gundara JS, Sidhu SB. Noncoding RNAs in endocrine malignancy. Oncologist 2014;19:483-491.

88. Modali SD, Parekh VI, Kebebew E, Agarwal SK. Epigenetic regulation of the lncRNA MEG3 and its target c-MET in pancreatic neuroendocrine tumors. Mol Endocrinol 2015;29:224-237.

89. Huarte M, Guttman M, Feldser D, et al. A large intergenic noncoding RNA induced by p53 mediates global gene repression in the p53 response. Cell 2010;142:409-419.

90. Hung T, Wang Y, Lin MF, et al. Extensive and coordinated transcription of noncoding RNAs within cell-cycle promoters. Nat Genet 2011;43:621-629.

91. Kung JT, Colognori D, Lee JT. Long noncoding RNAs: Past, present, and future. Genetics 2013;193:651-669.

92. Ballehaninna UK, Chamberlain RS. The clinical utility of serum CA 19-9 in the diagnosis, prognosis and management of pancreatic adenocarcinoma: An evidence based appraisal. J Gastrointest Oncol 2012;3:105-119.

93. Geary RS, Henry SP, Grillone LR. Fomivirsen: Clinical pharmacology and potential drug interactions. Clin Pharmacokinet 2002;41:255-260.

94. Lee RG, Crosby J, Baker BF, Graham MJ, Crooke RM. Antisense technology: An emerging platform for cardiovascular disease therapeutics. J Cardiovasc Transl Res 2013;6:969-980.

95. Zong X, Huang L, Tripathi V, et al. Knockdown of nuclearretained long noncoding RNAs using modified DNA antisense oligonucleotides, in nuclear bodies and noncoding RNAs: Methods and protocols, S. Nakagawa and T. Hirose (editors), Springer New York: New York, NY; 2015, pp. 321-331. 